\title{
Research on Measurement and Indication technique of Bolt Axis Force using Deformation of Bolt Shaft
}

\author{
Hiroyuki SASAGAWA*, Masanobu CHIBA**
}

Bolts have a significant impact the function of a wide range of mechanisms and often cause serious accident if applied incorrectly. Beyond lab tests, there has been no system available to accurately and cost effectively measure axial bolt loads during production and maintenance.

This paper deals with the development of such a system. Axial load will be investigated by measuring the deformation of a bolt with a shank reamed concentrically using the calculations and by measuring the displacement of a fluid in void of the shank to determine the change in volume.

Key Words: screw, bolt, axis force, fluid, safety

\section{I.Introduction}

Bolts play a large role in function of many machines and the loosened bolts often causes serious accidents. It is possible to measure the axial load acting on a bolt by measuring the elongation of the shank which is proportional to the load. This is most commonly done with strain gauges and supersonic wave axis power meters. Strain gages provide good results but are time consuming to use and cannot be used in real world situations. Sonic wave axis power meters are an option for taking measurements of the elongation in real time but are prohibitively expensive for routine use for those without large budgets.

Another option is to use tension indicating bolts which are calibrated to change color upon reaching a specified load but these bolts lack the ability to provide precise values.

\section{II.Axial Force and Axial Displacement}

The bolt with the shank reamed concentrically is shown in Fig.1.

\footnotetext{
*polytechnic University Japan

**polytechnic University Japan
}

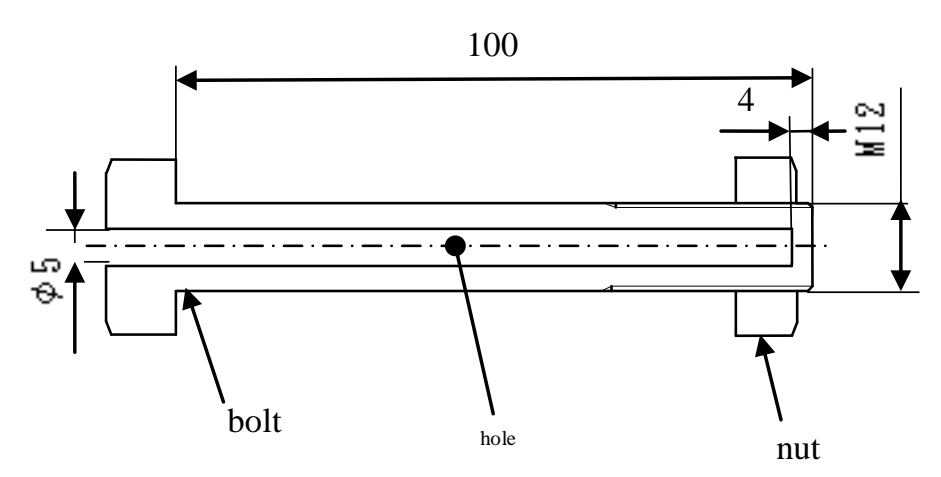

Fig.1 Analytical model of the bolt

When a tensile axial load $F$ is applied, the change in length $(\Delta L)$ is calculated as follows:

$$
\Lambda L=F \cdot L /(A \cdot E)
$$

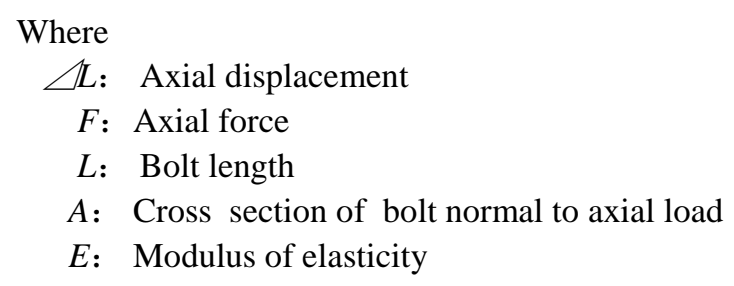

The Experimental value for $\angle L$ is obtained by attaching a shaft coaxially in the hole of the bolt and securing it to the tip to act as a reference point as shown Fig.2. An axial load F will bring the coaxial shaft away from being flush with the head of the bolt. This will allow for the elongation of the bolt to be measured. (Fig.3)

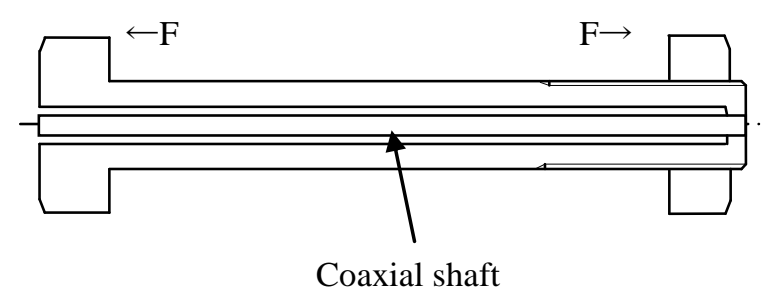

Fig.2 Coaxial shaft 
Proc. of The Third Intl. Conf. on Advances in Mechanical and Robotics Engineering - AMRE 2015 Copyright (C) Institute of Research Engineers and Doctors, USA .All rights reserved.

ISBN: 978-1-63248-066-8 doi: 10.15224/ 978-1-63248-066-8-61

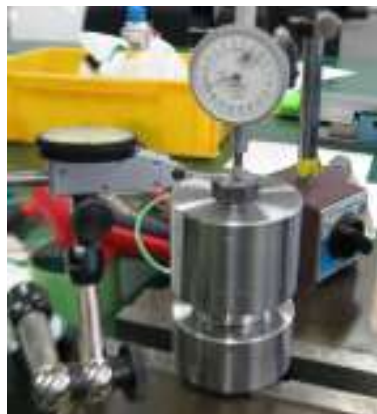

Fig.3 Measurement of displacement

The measurement value were in good agreement with the calculated values as seen in Fig.4.

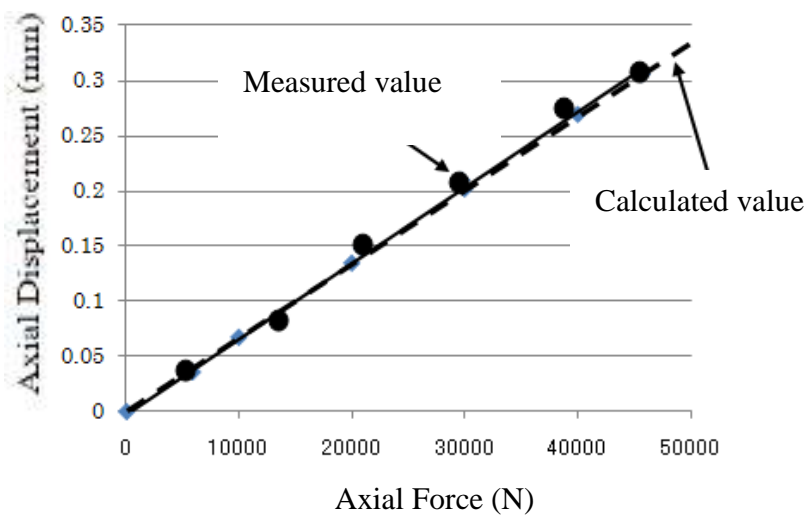

Fig.4 Comparison of calculated value and measured value

\section{The Relation between Axial Force and Radial Displacement}

The relationship between axial force and radial displacement of internal hole was analyzed using the finite element method software. Fig. 5 shows the bolt model of the bolt.

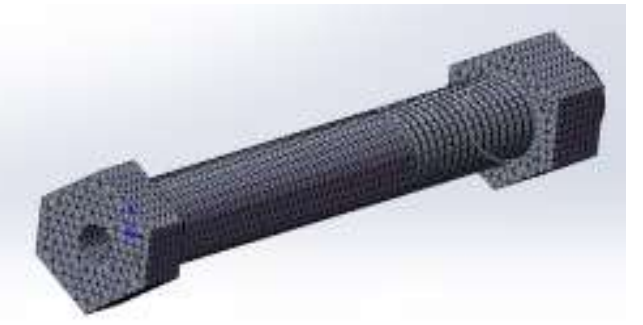

Fig.5 Analytical Model(FEM)

The plot of radial displacement versus axial force is shown in Fig.6. The axial force is determined by measuring the diameter of the hole.

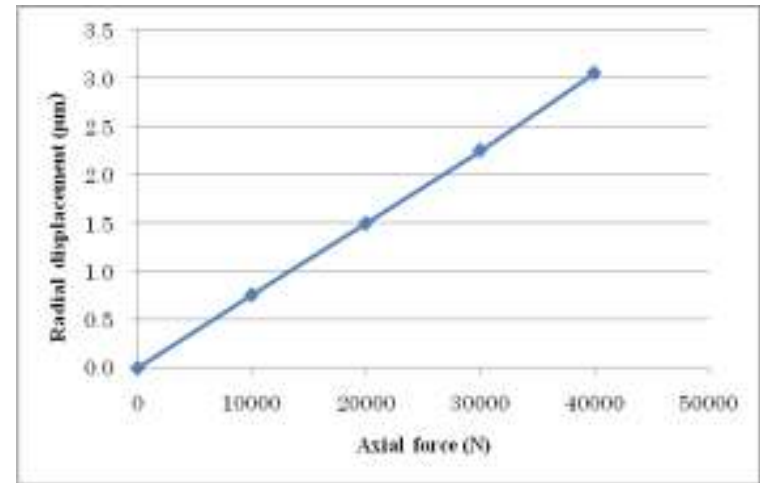

Fig.6 Radial displacement of internal hole

\section{Iv. The Relation between Axial Force and Change in Volume of Internal Hole}

An increasing axial load causes the diameter of internal hole to change proportionally with the rest of the bolt.i.e. the depth increases and the diameter decreases. This results in an increases in the total volume of the hole shown as follows.

$$
\Delta V=\Delta V_{1}-\Delta V_{2}
$$

Where

$\triangle V:$ Total change of volume

$\Delta V_{l}$ : Change in volume due to axial deformation

$\triangle V_{2}$ : Change in volume due to radial deformation

$\Delta \mathrm{V}$ is determined experimentally by measuring the displacement of the liquid in the internal pipe in Fig7. The change in dimensions of the hole is proportional to the axial force. The change of the level of the liquid is as follows:

\section{Lpipe $=$ N/Apipe}

Where

$\triangle$ Lpipe :displacement of liquid from reference point Apipe :cross-sectional area of pipe

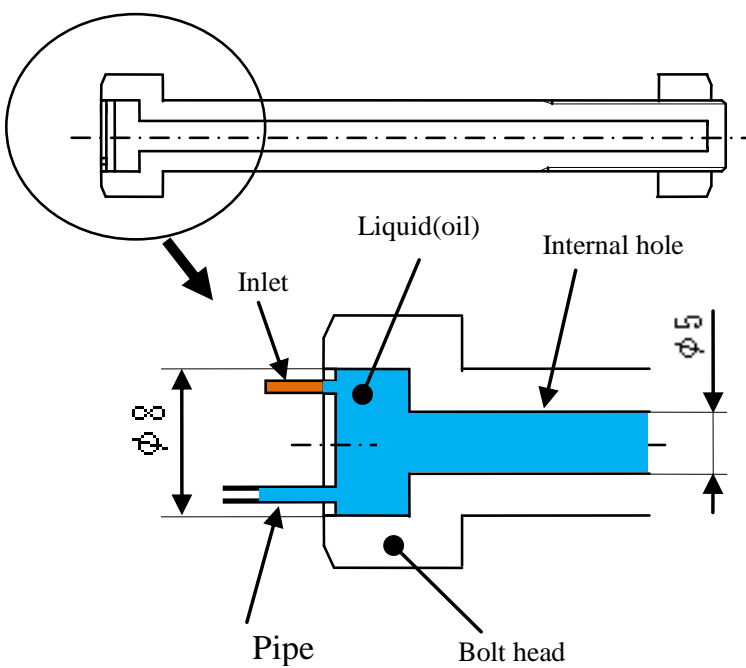

Fig.7 Structure of the bolt with the pipe 
Fig. 7 shows how the pipe was installed in the central hole to measure the change in volume. An increasing tensile axial load causes the volume of the internal hole to increase and the liquid level to drop. The liquid level is measured as shown as Fig.8. Using narrow pipe make ease to measure the level.

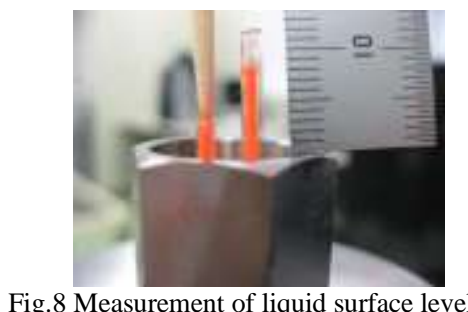

Plots of experimental measurements of the change of the liquid level is agreed well with the expected values Fig. 9.

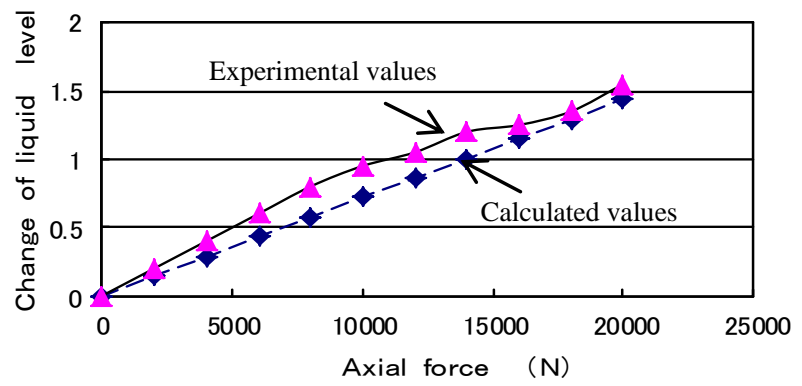

Fig.9 relation between calculated value and experimental value The liquid level indicator pipe may be piped and stored within the head of the bolt for practical situation.(Fig.10)

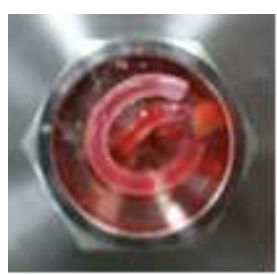

Fig.10 Pipe storage structure

\section{v. Conclusion}

The authors have devised a bolt design which enable precise, accurate and cost effective measurement of bolt tension in an uncomplicated manner. This device is also able to be housed within the bolt itself. This is achieved by a concentric hole along the length of the bolt .

And measuring the change in dimension of this hole in order to determine the axial load acting on the bolt.

\section{References}

[1] Akira Yamamoto, Principle and design of the screw fastening body, Youkendo ,1996

[2] Akira Yamamoto, A theory and calculation of the screw conclusion, Youkendo ,1970

[3] Isamu Yoshimoto, The main point of the screw fastening body design, Japan Standards Association,2002

[4] Yoshio Kuriyama, Hiroyuki Sasagawa, Mechanical design using CAE, Sankaido, 2005

[5] Hiroyuki Sasagawa, Masanobu Chiba, Research of Measurement Method of Axis Force using Displacement Expansion Mechanism with the Fluid ,Japan Society for Design Engineering,2014

[6] Hiroyuki Sasagawa, Msanobu Chiba, Research of Bolt Measurement Method of Axis Force using the Coaxial Shaft ,Japan Society for Design Engineering,2013 